\title{
High rates of transmitted NNRTI resistance among persons with acute HIV infection in Malawi: implications for first-line dolutegravir scale-up
}

\author{
Sarah E. Rutstein ${ }^{1 *}$, Jane S. Chen ${ }^{2}$, Julie A. E. Nelson ${ }^{1,3}$, Samuel Phiri ${ }^{4}$, William C. Miller ${ }^{5}$ \\ and Mina C. Hosseinipour ${ }^{1,6}$
}

\begin{abstract}
High rates of non-nucleoside reverse transcriptase inhibitors (NNRTI) resistance was a key consideration in the WHO policies transitioning first-line regimens to include integrase inhibitors (dolutegravir [DTG]). However, recent data suggests a relationship between DTG and neural tube defects among women exposed during conception, giving providers and policymakers pause regarding the planned regimen changes. We examined HIV drug resistance among a cohort of 46 acutely infected persons in Malawi. Our data demonstrates high levels of transmitted resistance, 11\% using standard resistance surveillance mutations and 20\% when additional NNRTI polymorphisms that may affect treatment response are included. High resistance rates in this treatment-naïve patient population reinforces the critical nature of DTG-based options in the context of public-health driven treatment programs.
\end{abstract}

Keywords: Antiretroviral resistance, Acute HIV infection, Malawi, Dolutegravir

\section{Background}

Current WHO-recommended first-line antiretroviral therapy (ART) regimens include non-nucleoside reverse transcriptase inhibitor (NNRTI) efavirenz in addition to two nucleoside analogue reverse transcriptase inhibitors [1]. However, increasing prevalence of NNRTI resistance among those initiating ART have raised concerns regarding the public health implications of efavirenz-based firstline regimens [2-6], as people with NNRTI-resistance are up to three times as likely to fail first-line therapy [7-11]. In response to escalating resistance, alternative firstline regimens may be critical to preserve morbidity and mortality benefits of early ART, as well as delay or avoid costly switch to second-line ART.

Dolutegravir (DTG) has been promoted as an alternative first-line ART backbone, with potential efficacy and

\footnotetext{
*Correspondence: srutstein@unc.edu

${ }^{1}$ Division of Infectious Diseases, University of North Carolina, Chapel Hill, NC, USA

Full list of author information is available at the end of the article
}

safety advantages over efavirenz and a very high barrier to resistance [12-14]. However, the logistical, cost, and clinical merits of such a policy shift depend directly on the underlying NNRTI-resistance patterns and prevalence. Furthermore, recent observational data from Botswana suggests a potential relationship between DTG and neural tube defects among women on DTG at time of conception has given many providers and policymakers pause regarding universal transitioning to a DTGbased backbone [15].

Here, we summarize NNRTI resistance patterns observed among treatment-naïve persons in Malawi identified with acute HIV infection (AHI). We include all mutation polymorphisms that potentially affect treatment response, as well as summarize mutations according to the WHO surveillance of drug resistance mutations list-widely accepted as those mutations most likely to represent transmitted drug resistance [16]. Our findings inform the ongoing conversation regarding risks and benefits of transitioning from efavirenz-based firstline to DTG-based first-line treatment regimens. 


\section{Analytical methods}

We analyzed resistance patterns from peripheral blood specimens collected at time of enrollment among participants in a pilot study evaluating behavioral and biomedical interventions for persons with AHI in Lilongwe, Malawi. Participants were screened and enrolled into the study between June 2012 and January 2014. Description of screening and study protocols are described elsewhere (clinicaltrials.gov \#NCT01450189) [17, 18]. AHI was defined as detectable HIV RNA with discordant or negative rapid HIV antibody tests. Where specimen volume allowed (39/46 specimens), repeat rapid antibody testing was conducted using rapid diagnostic assays (Determine $^{\mathrm{TM}}$ HIV 1/2 Antibody [Abbott Diagnostics, Illinois US] and Uni-Gold Recombigen ${ }^{\circledR}$ HIV 1/2 [Trinity Biotech, Ireland]) to confirm antibody negative/discordancy. We did not screen for receipt of pre or post-exposure prophylaxis; PrEP was not available in Malawi at the time of the study and although remote exposure to PEP was possible, PEP exposure since the very recent infection acquisition was considered unlikely given that screening represented the first presentation to care for this cohort of acutely infected persons. Additional eligibility criteria included: above definition of AHI within 21 days of enrollment, age $\geq 18$ years, and intention to remain in the Lilongwe area for the duration of study follow-up (52 weeks).

Integrase and reverse transcriptase (RT) genotypic resistance testing was performed for all participants using specimens collected at time of enrollment, which was on average 8 days after screening. All genotypic resistance testing was conducted on stored specimens after completion of study activities and thus was not available for real-time patient management. Reverse transcriptase population sequencing to determine resistance was performed as described previously [19]. After alignment using Sequencher (Gene Codes), resistance mutations were classified based on information in the Stanford University Drug Resistance Database (http:// hivdb.stanford.edu/index.html). We present both the full complement of resistance mutations as well as the resistance rates as limited to those identified by the WHO surveillance of drug resistance mutations list (SDRM) [16]. Sample integrity was checked by aligning the sequences using CLC Sequence Viewer (CLC bio A/S). Ninety-five percent confidence intervals $(\mathrm{CI})$ were calculated using exact methods in Stata (version 14.1, College Station, TX).

The National Health Sciences Research Committee of Malawi, the Malawi Medicines and Poisons Board, the Biomedical Institutional Review Board at University of North Carolina, Chapel Hill, and the National Institute of Allergy and Infectious Diseases' Prevention Science
Review Committee approved the procedures for this study. All study participants provided written informed consent in the local language or English, if preferred.

\section{Results and discussion}

Among 59 persons identified with AHI, 46 were enrolled into the study [20]. Of these 46 participants, 45 had resistance testing; nine $(20 \%$; $95 \%$ CI $10-35 \%)$ had at least one NNRTI resistance mutation detected (Table 1) and three had NRTI mutations detected (Table 2). There were no integrase mutations detected. Limiting likely transmitted drug resistance classification to those with mutations appearing in the SDRM list, five (11\%; 95\% CI 4-24\%) had NNRTI resistance; the most frequent mutation was $\mathrm{K} 103 \mathrm{~N}$, detected in four participants with any NNRTI mutations. The second most common mutation was E138A (3/9: 33\%), a mutation that is not on the SDRM but which nonetheless may be clinically significant in its effect on treatment response.

Table 1 Demographics and resistance outcomes

\begin{tabular}{|c|c|c|c|}
\hline & $\begin{array}{l}\text { Total }(n=46) \\
N(\%)\end{array}$ & $\begin{array}{l}\text { No } \\
\text { resistance } \\
(n=36) \\
N(\%)\end{array}$ & $\begin{array}{l}\text { Any } \\
\text { resistance } \\
(n=9) \\
N(\%)\end{array}$ \\
\hline \multicolumn{4}{|l|}{ Age } \\
\hline $18-24$ & $19(41)$ & $14(78)$ & $4(22)$ \\
\hline $25-34$ & $20(43)$ & $15(75)$ & $5(25)$ \\
\hline $35-44$ & $6(13)$ & $6(100)$ & $0(0)$ \\
\hline$\geq 45$ & $1(2)$ & $1(100)$ & $0(0)$ \\
\hline \multicolumn{4}{|l|}{ Sex } \\
\hline Male & $28(61)$ & $21(78)$ & $6(22)$ \\
\hline Female & $18(39)$ & $15(83)$ & $3(17)$ \\
\hline \multicolumn{4}{|l|}{ Marital status } \\
\hline Never married & $11(24)$ & & \\
\hline Married & $23(50)$ & & \\
\hline $\begin{array}{l}\text { Separated/divorced/wid- } \\
\text { owed }\end{array}$ & $12(26)$ & & \\
\hline \multicolumn{4}{|l|}{ Viral load at screening } \\
\hline$\leq 6 \log _{10}$ copies $/ \mathrm{mL}$ & $24(53)$ & $19(83)$ & $4(17)$ \\
\hline$>6 \log _{10}$ copies $/ \mathrm{mL}$ & $21(47)$ & $16(76)$ & $5(24)$ \\
\hline \multicolumn{4}{|c|}{ NNRTI resistance mutations frequency ${ }^{a}$} \\
\hline A98G & $2(4.4)$ & & \\
\hline E138A & $3(6.7)$ & & \\
\hline K101E & $1(2.2)$ & & \\
\hline $\mathrm{K} 103 \mathrm{~N}$ & $4(8.9)$ & & \\
\hline K103Q & $1(2.2)$ & & \\
\hline V179E & $1(2.2)$ & & \\
\hline V901 & $1(2.2)$ & & \\
\hline Y181C & $1(2.2)$ & & \\
\hline
\end{tabular}


Table 2 HIV drug resistance mutation profiles

\begin{tabular}{lll}
\hline Profile \# & NNRTI mutation & NRTI mutation \\
\hline 1 & A98G, K103N & M184V, T215F \\
2 & E138A $^{a}$ & \\
3 & E138A $^{a}$ & \\
4 & E138A & \\
5 & V179E & \\
5 & K103N & \\
6 & K103N & \\
7 & K103N & T69N \\
8 & K103Q $^{\text {a }}$ & K219R \\
9 & V901, A98G, K101E, Y181C & \\
10 & None & \\
\hline
\end{tabular}

NNRTI non-nucleoside reverse transcriptase inhibitors, NRTI nucleoside reverse transcriptase inhibitors, WHO World Health Organization

a Designated mutations are not on the WHO surveillance of drug resistance mutations list and thus may be less likely to represent transmitted drug resistance

Increasing levels of ART resistance are jeopardizing the success of ART scale-up, and specifically may compromise the efficacy and effectiveness of existing firstline, efavirenz-based ART regimens. WHO surveillance demonstrates steadily increasing resistance since 2001, particularly in southern and eastern Africa [2, 6]. Pretreatment drug resistance has previously been estimated upwards of $10 \%$ [6]; in our study, among persons with $\mathrm{AHI}$, transmitted drug resistance was identified in $11 \%$ (according to SDRM definitions) and 20\% of patients at least one NNRTI mutation. Observed rates are similar to those in recent WHO reports from Malawi, where 4/26 (15\%) of ARV drug-naïve persons had NNRTI mutations [6]. High resistance rates support the urgency of the DTG transition-a likely cost-effective programmatic shift of first-line ART regimen [21].

Vigilance is needed in monitoring response to therapy and any possible AE related to initiation of DTG. Ongoing evaluations include prospective clinical trials and observational studies focusing on several key populations (pregnant women, HIV-TB co-infection) [22]. Nonetheless, improved safety outcomes profiles, higher barriers to resistance, more favorable clinical tolerability, and cost-effectiveness modeling all suggest that DTG is a preferred first-line agent compared to efavirenz. Initiation of DTG in women of childbearing potential should be pursued cautiously, and with an informed provider and patient-population [15]. Our data demonstrates high levels of transmitted NNRTI resistance in Malawi, compromising the effectiveness EFV-based regimens. These data underscores the urgency of ongoing evaluations of the safest means by which to transition treatment initiation to DTG-based options. Persons with AHI represent a unique population for evaluation of transmitted drug resistance and similar evaluations may be warranted in other LMIC to better clarify implications of EFV-backbone as first-line therapy.

\begin{abstract}
Abbreviations
AE: adverse event; AHI: acute HIV infection; ART: antiretroviral therapy; DTG: Dolutegravir; EFV: efavirenz; LMIC: low- and middle-income countries; NNRTI: non-nucleoside reverse transcriptase inhibitors; NRTI: nucleoside reverse transcriptase inhibitors; RT: reverse transcriptase; SDRM: surveillance of drug resistance mutations; WHO: World Health Organization.
\end{abstract}

\section{Authors' contributions}

SER and MCH drafted the initial manuscript. SER, SP, WCM and MCH were all investigators on the primary clinical trial that produced this data. JSC conducted data analysis and contributed to drafting. JAEN conducted all resistance assays and analyses. All authors read and approved the final manuscript

\section{Author details}

${ }^{1}$ Division of Infectious Diseases, University of North Carolina, Chapel Hill, NC, USA. ${ }^{2}$ Department of Epidemiology, University of North Carolina, Chapel Hill, NC, USA. ${ }^{3}$ Department of Microbiology and Immunology, University of North Carolina, Chapel Hill, NC, USA. ${ }^{4}$ Lighthouse Trust, Lilongwe, Malawi. ${ }^{5}$ Department of Epidemiology, Ohio State University, Columbus, OH, USA. ${ }^{6}$ UNC Project, Lilongwe, Malawi.

\section{Acknowledgements}

The authors acknowledge the numerous HIV testing and counseling staff at Lighthouse and UNC Project clinics who assisted in study activities, as well as the patients who contributed their time as participants in this study.

\section{Competing interests}

The authors declare that they have no competing interests.

\section{Availability of data and materials}

The data that support the findings of this study are available from National health Sciences Research Committee of Malawi and the Biomedical Institutional Review Board of University of North Carolina via the corresponding author, but restrictions apply to the availability of these data, which were used under license for the current study, and so are not publicly available. Data are however available from the authors upon reasonable request and with permission of above named ethic boards.

\section{Consent for publication}

Not applicable.

\section{Ethics approval and consent to participate}

The National Health Sciences Research Committee of Malawi, the Malawi Medicines and Poisons Board, the Biomedical Institutional Review Board at University of North Carolina, Chapel Hill, and the National Institute of Allergy and Infectious Diseases' Prevention Science Review Committee approved the procedures for this study. All study participants provided written informed consent in the local language or English, if preferred.

\section{Funding}

This work was supported by National Institutes of Health, National Institute of Allergy and Infectious Diseases [R01 Al083059]. S.E.R. was supported by National Institutes of Health, National Institute of Mental Health [F30 MH098731]. JSC was supported by National Institutes of Health, National Institute of Allergy and Infectious Diseases [T32 Al070114].

\section{Publisher's Note}

Springer Nature remains neutral with regard to jurisdictional claims in published maps and institutional affiliations. 
Received: 28 November 2018 Accepted: 7 February 2019

Published online: 22 February 2019

\section{References}

1. WHO. Consolidated guidelines on the use of antiretroviral drugs for treating and preventing HIV infection: recommendations for a public health approach. 2nd ed. Geneva: World Health Organization; 2016.

2. Gupta RK, Gregson J, Parkin N, Haile-Selassie H, Tanuri A, Andrade Forero $\mathrm{L}$, et al. HIV-1 drug resistance before initiation or re-initiation of first-line antiretroviral therapy in low-income and middle-income countries: a systematic review and meta-regression analysis. Lancet Infect Dis. 2018;18(3):346-55.

3. Hong SY, Jonas A, DeKlerk M, Shiningavamwe A, Desta T, Badi A, et al. Population-based surveillance of HIV drug resistance emerging on treatment and associated factors at sentinel antiretroviral therapy sites in Namibia. J Acquir Immune Defic Syndr. 2015;68(4):463-71.

4. Mungati M, Mhangara M, Gonese E, Mugurungi O, Dzangare J, Ngwende $\mathrm{S}$, et al. Pre-treatment drug resistance among patients initiating antiretroviral therapy (ART) in Zimbabwe: 2008-2010. BMC Res Notes. 2016;9:302.

5. Rowley CF, MacLeod IJ, Maruapula D, Lekoko B, Gaseitsiwe S, Mine M, et al. Sharp increase in rates of HIV transmitted drug resistance at antenatal clinics in Botswana demonstrates the need for routine surveillance. J Antimicrob Chemother. 2016;71(5):1361-6.

6. World Health Organization. HIV drug resistance report 2017. Geneva: World Health Organization; 2017.

7. Avila-Rios S, Garcia-Morales C, Matias-Florentino M, Romero-Mora KA, Tapia-Trejo D, Quiroz-Morales VS, et al. Pretreatment HIV-drug resistance in Mexico and its impact on the effectiveness of first-line antiretroviral therapy: a nationally representative 2015 WHO survey. Lancet HIV. 2016;3(12):e579-91.

8. Hamers RL, Schuurman R, Sigaloff KC, Wallis CL, Kityo C, Siwale M, et al. Effect of pretreatment HIV-1 drug resistance on immunological, virologi$\mathrm{cal}$, and drug-resistance outcomes of first-line antiretroviral treatment in sub-Saharan Africa: a multicentre cohort study. Lancet Infect Dis. 2012;12(4):307-17.

9. Kantor R, Smeaton L, Vardhanabhuti S, Hudelson SE, Wallis CL, Tripathy $\mathrm{S}$, et al. Pretreatment HIV drug resistance and HIV-1 subtype $C$ are independently associated with virologic failure: results from the multinational PEARLS (ACTG A5175) clinical trial. Clin Infect Dis. 2015;60(10):1541-9.

10. Wittkop L, Gunthard HF, de Wolf F, Dunn D, Cozzi-Lepri A, de Luca A, et al. Effect of transmitted drug resistance on virological and immunological response to initial combination antiretroviral therapy for HIV (EuroCoordCHAIN joint project): a European multicohort study. Lancet Infect Dis. 2011:11(5):363-71.
11. World Health Organization. Guidelines on the public health response to pretreatment HIV drug resistance. Geneva: World Health Organization; 2017.

12. Walmsley S, Baumgarten A, Berenguer J, Felizarta F, Florence E, KhuongJosses MA, et al. Brief report: dolutegravir plus abacavir/lamivudine for the treatment of HIV-1 infection in antiretroviral therapy-naive patients: week 96 and week 144 results from the SINGLE randomized clinical trial. J Acquir Immune Defic Syndr. 2015;70(5):515-9.

13. Walmsley SL, Antela A, Clumeck N, Duiculescu D, Eberhard A, Gutierrez F, et al. Dolutegravir plus abacavir-lamivudine for the treatment of HIV-1 infection. N Engl J Med. 2013;369(19):1807-18.

14. Zash R, Jacobson DL, Diseko M, Mayondi G, Mmalane M, Essex M, et al. Comparative safety of dolutegravir-based or efavirenz-based antiretroviral treatment started during pregnancy in Botswana: an observational study. Lancet Glob Health. 2018;6(7):e804-10.

15. Zash R, Makhema J, Shapiro RL. Neural-tube defects with dolutegravir treatment from the time of conception. N Engl J Med. 2018;379(10):979-81.

16. Bennett DE, Camacho RJ, Otelea D, Kuritzkes DR, Fleury H, Kiuchi M, et al. Drug resistance mutations for surveillance of transmitted HIV-1 drugresistance: 2009 update. PLoS ONE. 2009;4(3):e4724.

17. Rutstein SE, Pettifor AE, Phiri S, Kamanga G, Hoffman IF, Hosseinipour MC, et al. Incorporating acute HIV screening into routine HIV testing at sexually transmitted infection clinics, and HIV testing and counseling centers in Lilongwe, Malawi. J Acquir Immune Defic Syndr. 2016;71(3):272-80.

18. Rucinski KB, Rutstein SE, Powers KA, Pasquale DK, Dennis AM, Phiri S, et al. Sustained sexual behavior change following acute HIV diagnosis in Malawi. Sex Transm Dis. 2018;45:741-6.

19. Farr SL, Nelson JA, Ng'ombe TJ, et al; BAN Study Team. Addition of 7 days of zidovudine plus lamivudine to peripartum single-dose nevirapine effectively reduces nevirapine resistance postpartum in HIV-infected mothers in Malawi. J Acquir Immune Defic Syndr. 2010;54:515-23.

20. Miller WC, Rutstein SE, Phiri S, Kamanga G, Nsona D, Pasquale DK, et al. Randomized controlled pilot study of antiretrovirals and a behavioral intervention for persons with acute HIV infection: opportunity for interrupting transmission. Open Forum Infect Dis. 2019;6(1):341.

21. Phillips AN, Cambiano V, Nakagawa F, Revill P, Jordan MR, Hallett TB, et al. Cost-effectiveness of public-health policy options in the presence of pretreatment NNRTI drug resistance in sub-Saharan Africa: a modelling study. Lancet HIV. 2018;5(3):e146-54.

22. Vitoria M, Hill A, Ford N, Doherty M, Clayden P, Venter F, et al. The transition to dolutegravir and other new antiretrovirals in low-income and middle-income countries: what are the issues? AIDS. 2018;32(12):1551-61.
Ready to submit your research? Choose BMC and benefit from:

- fast, convenient online submission

- thorough peer review by experienced researchers in your field

- rapid publication on acceptance

- support for research data, including large and complex data types

- gold Open Access which fosters wider collaboration and increased citations

- maximum visibility for your research: over $100 \mathrm{M}$ website views per year

At BMC, research is always in progress.

Learn more biomedcentral.com/submissions 